\title{
COVID-19 Pandemic Control Measures: Impact on Social Frailty and Health Outcomes in Non-Frail Community-Dwelling Older Adults
}

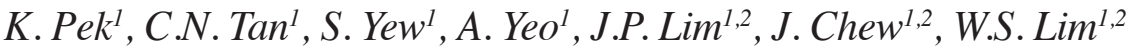 \\ 1. Institute of Geriatrics and Active Ageing, Tan Tock Seng Hospital, Singapore; 2. Department of Geriatric Medicine, Tan Tock Seng Hospital, Singapore \\ Corresponding Author: Kalene Pek, Tan Tock Seng Hospital, Singapore, kalene_sl_pek@ttsh.com.sg
}

Dear Editor,

$\mathrm{M}$ ore than one year on, countries continue to impose varying intensities of public health measures to curb the COVID-19 pandemic, running the gamut from encouragement to stay home to the other spectrum of stringent lockdown measures. Older adults remain most vulnerable to both direct and indirect impact of COVID-19; specifically, secondary effects of pandemic control measures such as access to health services and social isolation remain to be determined (1). For instance, an audit study highlighted the disruption of essential community support services for older adults with dementia, leading to behavioral changes that necessitated titration of psychotropic medications and increased caregiver stress (2).

Since the first case detected on January 23, 2020, the Singapore government has responded with calibrated COVID19 measures to mitigate the virus transmission. This included raising the Disease Outbreak Response System Condition (DORSCON) level to Orange (moderate) since February 7, 2020 (3), and implementing an 8-week circuit breaker (CB) from April 7 to June 1, 2020, where only essential services remained available with severe reduction of eldercare services (4). Beyond the CB, a three-phased approach ensured activities were resumed progressively, including support and activities for older adults, from June 2, 2020 onwards.

While recent studies highlighted the buffering effect of social relationships against negative changes in older adults' psychological well-being due to COVID-19 measures (5), the impact on social frailty is less well understood, possibly due to challenges of conducting such studies in a time-sensitive manner. This provided the impetus for the current study, which involves secondary analysis of an ongoing longitudinal cohort study to ascertain the impact of pandemic control measures on social frailty and health outcomes among healthy communitydwelling older adults. The accrued understanding can help guide policy planning in mitigating secondary effects of pandemic control measures on older adults.

We studied 203 participants from the "Longitudinal Assessment of Biomarkers for characterization of early Sarcopenia and Osteosarcopenic Obesity in predicting frailty and functional decline in community-dwelling Asian older adults study" (GeriLABS-2) (6) who completed one-year follow-up visit. One hundred and fifty-one (74.3\%) completed face-to-face assessment prior to DORSCON Orange enhanced Received March 26, 2021

Accepted for publication April 6, 2021 pandemic control measures (termed 'pre-circuit breaker' group), while fifty-two (25.6\%) were assessed remotely between July to August 2020 ('post-circuit breaker' group) after control measures were gradually lifted. Ethics amendment approval was obtained for remote assessment using modified data collection that cohered with control measures.

The primary outcome measure was social frailty, measured using the validated 8-item social frailty scale (SFS-8, range: 0-8) with higher scores indicating increased social frailty (7). The SFS- 8 has a three-factor structure comprising 'social resources' (Factor-1), 'social activities and financial resource' (Factor-2), and 'social need fulfilment' (Factor-3). Secondary outcomes included Chinese Mini-Mental State Examination (CMMSE); 15-item Geriatric Depression Scale (GDS); basic and instrumental activities of daily living (ADLs); FRAIL scale; lifestyle activity (Frenchay Activities Index, FAI); and physical activity (International Physical Activity Questionnaire, IPAQ).

We performed intra-group comparison between baseline and one-year assessment for both pre- and post-CB groups using paired T-test. To account for baseline difference in age between pre- and post-CB groups (68.07 \pm 7.37 vs $63.62 \pm 6.39$ years, $\mathrm{p}<.001)$, we performed linear regression adjusting for age and gender for inter-group comparison of one-year outcomes, with significance set at $5 \%$.

Participants were predominantly Chinese and female. They were cognitively intact, non-frail by FRAIL $(<3)$ criteria and independent in basic and instrumental ADLs (Table 1), attesting to their relatively good health. In the pre-CB intragroup comparison, social frailty total and factor scores were significantly improved at one-year, while other outcomes did not differ. The post-CB intra-group comparison showed significant increase in social frailty total score $(0.73 \pm 0.95$ vs $1.85 \pm 1.16, \mathrm{p}<.001)$. Underpinned by increase in factors 1 and 2 , the total score approached the cutoff $(\geq 2)$ for social prefrailty, which previously was reported to be highly associated with lower physical activity and poorer physical performance (7). Both GDS and FAI scores were significantly poorer at one-year, although CMMSE, ADLs and IPAQ did not differ. Inter-group comparison at one-year revealed post-CB group being significantly worse in social frailty total as well as factor 1 and 2 scores, whereas factor 3 was paradoxically better. For secondary outcomes, GDS and FAI were significantly worse in post-CB group, but not different in CMMSE, ADLs and IPAQ.

In summary, our study shed light on the deleterious impact 
Table 1. Inter- and intra-group comparisons for pre- and post-circuit breaker assessments

\begin{tabular}{|c|c|c|c|c|}
\hline & \multicolumn{2}{|c|}{ Pre-Circuit Breaker $(\mathrm{n}=151)$} & \multicolumn{2}{|c|}{ Post-Circuit Breaker $(\mathrm{n}=52)$} \\
\hline & Baseline & 1-year & Baseline & 1-year \\
\hline \multicolumn{5}{|l|}{ Demographics } \\
\hline Gender, female, $\mathrm{n}(\%)$ & $110(72.8)$ & & $40(76.9)$ & \\
\hline Race, Chinese, n (\%) & $140(92.7)$ & & $50(96.2)$ & \\
\hline \multicolumn{5}{|l|}{ Cognitive performance } \\
\hline \multicolumn{5}{|l|}{ Mood } \\
\hline GDS, max 15 & $1.03 \pm 1.44$ & $1.05 \pm 1.33$ & $1.19 \pm 1.78$ & $1.92 \pm 1.73^{\mathrm{a}, \mathrm{b}}$ \\
\hline \multicolumn{5}{|l|}{ Frailty status } \\
\hline FRAIL score, $\max 5$ & $0.19 \pm 0.44$ & $0.27 \pm 0.59$ & $0.12 \pm 0.32$ & $0.08 \pm 0.27$ \\
\hline \multicolumn{5}{|l|}{ Activity } \\
\hline FAI, $\max 40$ & $32.16 \pm 5.31$ & $32.27 \pm 5.28$ & $33.37 \pm 3.96$ & $30.04 \pm 5.47^{\mathrm{a}, \mathrm{b}}$ \\
\hline IPAQ, METs & $5199.25 \pm 2384.81$ & $5125.06 \pm 2131.19$ & $4594.81 \pm 2254.75$ & $4849.58 \pm 2603.80$ \\
\hline \multicolumn{5}{|l|}{ Social frailty, SFS-8\# } \\
\hline Total score, $\max 8$ & $1.39 \pm 1.42$ & $0.89 \pm 1.15^{\mathrm{a}}$ & $0.73 \pm 0.95$ & $1.85 \pm 1.16^{\mathrm{a}, \mathrm{b}}$ \\
\hline Factor 1: Social resources, $\max 3$ & $0.46 \pm 0.77$ & $0.22 \pm 0.58^{\mathrm{a}}$ & $0.19 \pm 0.60$ & $0.67 \pm 0.73^{\mathrm{a}, \mathrm{b}}$ \\
\hline Factor 2: Social activities \& financial resource, $\max 3$ & $0.51 \pm 0.76$ & $0.36 \pm 0.58^{\mathrm{a}}$ & $0.35 \pm 0.59$ & $1.10 \pm 0.63^{\mathrm{a}, \mathrm{b}}$ \\
\hline Factor 3: Social need fulfilment, $\max 2$ & $0.42 \pm 0.59$ & $0.31 \pm 0.57^{\mathrm{a}}$ & $0.19 \pm 0.49$ & $0.10 \pm 0.30^{\mathrm{b}}$ \\
\hline
\end{tabular}

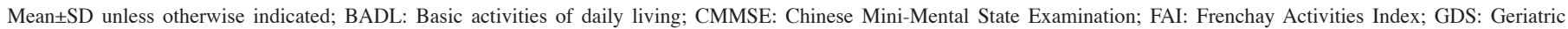

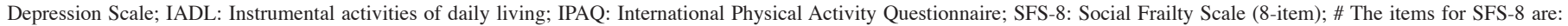

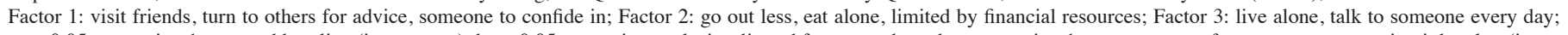

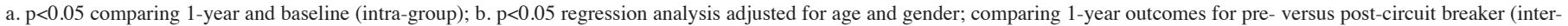
group).

of COVID-19 pandemic control measures on social frailty and attendant health outcomes in non-frail community-dwelling older adults. Even though post-CB participants were less socially frail at baseline compared to pre-CB counterparts, they endorsed significantly increased social frailty which was driven by impact on social resources, social activities and financial resource. Further studies are warranted to evaluate if the observed improvement in social need fulfilment at oneyear can be attributable to other factors such as work-fromhome arrangements, home-based learning, or differences in digital literacy. Notably, even though cognition, ADLs and physical activity were relatively preserved in our cohort of nonfrail older adults, the impact on social frailty was associated with poorer mood and decreased lifestyle activity. The greater depressive symptoms observed in the post-CB group echoed previous findings (8), whereas decreased lifestyle activity as a result of pandemic control measures was unsurprising, given that FAI measured higher order ADLs that included more complex social activities beyond physical activity (9). Enabling social engagement and connectedness for older adults is therefore an imperative that cannot be compromised (10).

One study limitation was the opportunistic nature of our data from an ongoing study, as opposed to a pre-planned study examining the impact of COVID-19 control measures.
Furthermore, being conducted in a non-frail Asian cohort that is predominantly Chinese, these results may not be generalizable to frail older adults and other ethnicities. Taken together, nonetheless, our study highlights that the impact of COVID-19 control measures on social frailty, mood and lifestyle activity extends even to relatively healthy older adults with implications for planning and implementation of future pandemic control policies. While COVID-19 has aggressively compelled us to change the way we live and connect socially, it also presents "a unique opportunity for improvement" (11) to enhance social support for our older adults and maintain preparedness for the post-pandemic epoch.

Ethial standards: Ethics approval for the study was obtained from the Domain Specific Review Board of the National Healthcare Group and informed written consent was obtained from the participants.

Conflict of interest: The authors have no conflicts of interest to disclose.

\section{References}

1. Lim WS, Liang CK, Assantachai P, Auyeung TW, Kang L, Lee WJ, et al. COVID-19 and older people in Asia: Asian Working Group for Sarcopenia calls to action. Geriatr Gerontol Int. 2020;20(6):547-58.

2. Koh ZY, Law F, Chew J, Ali N, Lim WS. Impact of Coronavirus Disease on Persons with Dementia and Their Caregivers: An Audit Study. Ann Geriatr Med Res 2020;24(4):316-20.

3. Channel NewsAsia. Coronavirus outbreak: Singapore raises DORSCON level 
to Orange; schools to suspend inter-school, external activities. https://www. channelnewsasia.com/news/singapore/wuhan-coronavirus-dorscon-orange-singaporerisk-assessment-12405180. Assessed 16 Mar 2021.

4. Ministry of Health. Updates on COVID-19 (Coronavirus Disease 2019) Local Situation. https://www.moh.gov.sg/covid-19. Assessed 16 Mar 2021.

5. Macdonald B, Hülür G. Well-Being and Loneliness in Swiss Older Adults During the COVID-19 Pandemic: The Role of Social Relationships. Gerontologist. 2021;61(2):240-50.

6. Lim WS, Lim JP, Chew J, Tan AWK. Influence of Obesity on Diagnostic Accuracy and Optimal Cutoffs for Sarcopenia Screening in Non-Frail Older Adults: A Comparison of SARC-F Versus SARC-CalF. J Nutr Health Aging. 2020;24(8):914-6.

7. Pek K, Chew J, Lim JP, Yew S, Tan CN, Yeo A, et al. Social Frailty Is Independently Associated with Mood, Nutrition, Physical Performance, and Physical Activity: Insights from a Theory-Guided Approach. Int J Environ Res Public Health. 2020;17(12):4239.
8. Robb CE, de Jager CA, Ahmadi-Abhari S, Giannakopoulou P, Udeh-Momoh C, McKeand J, et al. Associations of Social Isolation with Anxiety and Depression During the Early COVID-19 Pandemic: A Survey of Older Adults in London, UK. Front Psychiatry. 2020;11:591120.

9. Turnbull JC, Kersten P, Habib M, McLellan L, Mullee MA, George S. Validation of the Frenchay Activities Index in a general population aged 16 years and older. Arch Phys Med Rehabil. 2000;81(8):1034-8.

10. Batsis JA, Daniel K, Eckstrom E, Goldlist K, Kusz H, Lane D, et al. Promoting Healthy Aging During COVID-19. J Am Geriatr Soc. 2021;69(3):572-80.

11. Cesari M. Proietti M. Geriatric Medicine in Italy in the Time of COVID-19. J Nutr Health Aging. 2020;24:459-60. 